an abnormal jejunal biopsy specimen. Both responded well to treatment.

The stools of five contacts of the second patient and of four other Pakistanis have been examined and hookworm infestation has been found in five of them.

It is suggested that a public-health problem exists on two counts. First, there may be many immigrants suffering from hookworm infestation who need to be treated for the sake of their own health. Secondly, the possibility of the spread of hookworm disease in Great Britain must exist while such cases go untreated.

\section{REFERENCES}

Adams, A. R. D., and Maegraith, B. G. (1960). Clinical Tropical Diseases, 2nd ed. Blackwell, Oxford.

Blacklock, D. B., and Southwell, T. (1961). A Guide to Human Parasitology, 7th ed. Lewis, London.

Foy, H., and Kondi, A. (1960). Trans. roy. Soc. trop. Med. Hyg., 54, 419.

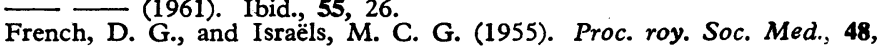
347.

Miller, G A. H and Bamforth J. (1962). Brit, med 7. 1, 1661.

Sheehy, T. W., Meroney, W. H., Cox, R. S., and Soler, J. E. (1962). Gastroenterology, 42, 148.

World Health Organization (1959). Tech. Rep. Ser., No. 182, p. 1.

\title{
Prevention of Poliomyelitis in Singapore by Live Vaccine
}

\author{
L. H. LEE,* M.B., B.S. ; K. A. LIM,* M.B., CH.B., B.SC. ; C. Y. TYE,* B.A.
}

Brit. med. F., 1964, 1, 1077-1080

In recent years Singapore's population has grown rapidly, accompanied by great improvement in general health standards. These changes are reflected in the data in Table $I$. Three-quarters of the population are Chinese, about $14 \%$ Malay, $8 \%$ Indian and Pakistani, and the remaining $3 \%$ are roughly equally divided between three groups-Eurasians, Europeans, and others.

TABLE I.-Trends in Some Vital Indices for Singapore, 1940-62

\begin{tabular}{c|c|c|c}
\hline & Population & $\begin{array}{c}\text { Crude Death Rate } \\
\text { per 1,000 Population }\end{array}$ & $\begin{array}{c}\text { Infant Mortality Rate } \\
\text { per 1,000 Live Births }\end{array}$ \\
\hline 1940 & 751,000 & $20 \cdot 9$ & $142 \cdot 6$ \\
1950 & $1,02,000$ & $12 \cdot 0$ & $82 \cdot 2$ \\
1960 & $1,64,000$ & $6 \cdot 2$ & $34 \cdot 9$ \\
1962 & $1,733,000$ & $5 \cdot 9$ & $31 \cdot 2$ \\
\hline
\end{tabular}

In recent years, too, the epidemiological pattern of poliomyelitis in Singapore has tended to show some transition from the endemic behaviour characteristic of countries with low health standards towards the epidemic behaviour characteristic of countries with high sanitary standards. However, cases continue to be confined predominantly to very young children, and the pattern is that of an endemic disease periodically breaking out in epidemic form. Such epidemic waves were seen in 1946 and 1948, soon after the war, but they subsequently appeared to be decreasing in violence until 1958, when an outbreak of over 400 cases occurred (see Table II).

TABLE II.-Notifications of Poliomyelitis in Singapore*

\begin{tabular}{c|c|c|c|c|c|c|c}
\hline Year & Cases & Year & Cases & Year & Cases & Year & Cases \\
\hline 1946 & 188 & 1951 & 94 & 1955 & 25 & 1959 & 62 \\
1947 & 0 & 1952 & 56 & 1956 & 72 & 1960 & 196 \\
1948 & 172 & 1953 & 47 & 1957 & 64 & 1961 & 53 \\
1949 & 78 & 1954 & 85 & 1958 & 415 & 1962 & 14 \\
1950 & 90 & & & & & & \\
\hline
\end{tabular}

* Non-paralytic cases rarely get to be notified, and the notification data therefore refer essentially to paralytic cases only.

During this epidemic, which was almost exclusively due to type 1 virus, an attempt was made to modify its course by wide-scale administration of type 2 attenuated virus (Sabin). This has been reported by Hale et al. (1959).

In 1959 the Singapore Government appointed a committee $^{1}$ to study the problems relating to the prevention of poliomyelitis in Singapore. In 1960 this committee carried out a serological and faecal survey and reported its findings to the Ministry of Health, Singapore (1961). In 1962 a mass immunization campaign was inaugurated, during which about $60 \%$ of children from 1 to 5 years of age received two doses of trivalent attenuated virus vaccine. In 1963 a routine programme was inaugurated whereby attenuated vaccine was given to young infants and to children first entering school.

This paper reports the investigations undertaken in the course of the committee's deliberations and the results to date of poliomyelitis immunization in Singapore.

\section{Survey}

This survey was designed to assess the immune status of children in Singapore and to study the results of the administration of type 2 vaccine in 1958-9.

Blood and stool specimens were taken from children below 5 years of age, this being the age-group principally effected by poliomyelitis in Singapore. The sample was drawn from healthy children attending at maternal and child health centres in both urban and rural areas. For the sake of homogeneity the survey was restricted to Chinese children. About $75 \%$ of the population at all ages are Chinese; therefore if other ethnic groups had been included their numbers would have been insufficient for comparative purposes unless the total sample size were greatly increased.

In the selection of subjects, those who had received Salk vaccine were excluded. Those who had documentary evidence or gave the date and time of receiving Sabin vaccine (type 2) during the previous campaign were classified as "vaccinated." Those who claimed never to have been vaccinated against poliomyelitis were classified as " nonvaccinated." In the latter group it was also ascertained that no other members of their household had been vaccinated. Response to the survey was voluntary and co-operation of the people was excellent.

By the time administrative difficulties had been overcome and the nature of the project explained to prospective sub-

* From the W.H.O. Regional Enterovirus Laboratory (formerly W.H.O. poliomyelitis centre in Singapore), Department of Bacteriology, University of Singapore and Department of Social Medicine and Public vealth, University of Singapore.

1 Government Committee on Poliomyelitis: Dr. Ng See Yook (chairman), Dr. K. Kanagaratnam, Dr. A. L. Gwee, Mr. C. Y. Tye, and Dr. L. H. Lee. Professor K. A. Lim was invited to advise the Committee. 
jects, it was already March 1960, some 18 months after the vaccination campaign of 1958 . For this reason there were no vaccinated children under 1 year of age and comparatively few even among the 1-year-olds. The composition of the 651 children sampled is shown in Table III.

TABLE III.-Distribution of Subjects by Age, Area, and Vaccination Categories. 1960 Survey

\begin{tabular}{|c|c|c|c|c|c|}
\hline \multirow{2}{*}{\multicolumn{2}{|c|}{ Age }} & \multicolumn{2}{|c|}{ City } & \multicolumn{2}{|c|}{ Rural } \\
\hline & & Vaccinated & $\underset{\text { Vaccinated }}{\text { Not }}$ & Vaccinated & $\underset{\text { Vaccinated }}{\text { Not }}$ \\
\hline 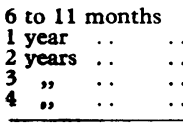 & $\begin{array}{l}. . \\
\because \\
\ddot{0}\end{array}$ & $\begin{array}{l}\overline{24} \\
54 \\
43 \\
22 \\
\end{array}$ & $\begin{array}{l}54 \\
64 \\
50 \\
38 \\
24 \\
\end{array}$ & $\begin{array}{l}\overline{18} \\
43 \\
29 \\
22 \\
\end{array}$ & $\begin{array}{l}43 \\
42 \\
41 \\
24 \\
16 \\
\end{array}$ \\
\hline Total . & .. & 143 & 230 & 112 & 166 \\
\hline
\end{tabular}

\section{Laboratory Methods}

Neutralization Tests.-Sera were separated from blood samples the day they were obtained and stored frozen until tested. A one-in-three dilution of serum was used for detecting neutralizing antibodies against 100 T.C.D. 50 of each of the three types of poliovirus. The three prototype viruses used were Mahoney, Lansing, and Leon. Serum-virus mixtures were incubated at $37^{\circ} \mathrm{C}$. for two hours, then held overnight at $4^{\circ} \mathrm{C}$., and again at $37^{\circ} \mathrm{C}$. for one hour before inoculation into monkey-kidney culture tubes. Four tubes were inoculated, and a serum was regarded as positive if it prevented cytopathogenicity of virus in at least two tubes at the end of four days.

Virus Isolation.-Specimens of faeces were processed within one day of collection. A $10 \%$ suspension was prepared in distilled water containing antibiotics and clarified by centrifugation at 10,000 r.p.m. for 30 minutes. The supernatant was stored frozen until inoculated into monkey-kidneytissue cultures. Cultures that were negative after 12 days were discarded. Cytopathogenic agents recovered were identified by neutralization tests against antisera for polioviruses and Coxsackie B types 1 to 5 and Coxsackie A9. As antisera for other enteroviruses were not available, those viruses that could not be identified were grouped together as "unclassified."

\section{Results}

The results of the serological survey are presented in Table IV and the faecal survey in Table V.

It is necessary to emphasize that the description " vaccinees" refers to children who had received Sabin type 2 vaccine in 1958. Thus the only direct effect of vaccination that might be expected would be the presence of type 2 antibody in vaccinees.

TABLE IV.-Percentages of Children with Neutralizing Antibody for
Poliovirus. Survey of 1960 , Singapore

\begin{tabular}{|c|c|c|c|c|c|c|}
\hline \multirow{2}{*}{$\begin{array}{l}\text { Age } \\
\text { in } \\
\text { Years }\end{array}$} & \multicolumn{2}{|c|}{ Type 1} & \multicolumn{2}{|c|}{ Type 2} & \multicolumn{2}{|c|}{ Type 3} \\
\hline & Vac. & Non-Vac. & Vac. & Non-Vac. & Vac. & Non-Vac. \\
\hline $\begin{array}{l}0^{*} \\
1 \\
2 \\
3 \\
4\end{array}$ & $\begin{array}{l}2 \overline{25 \cdot 0} \\
42 \cdot 6 \\
55 \cdot 8 \\
54 \cdot 5\end{array}$ & $\begin{array}{r}5 \cdot 6 \\
9 \cdot 4 \\
42 \cdot 0 \\
71 \cdot 1 \\
75 \cdot 0\end{array}$ & $\begin{array}{r}\text { City } \\
-1 \cdot 5 \\
87 \cdot 0 \\
90 \cdot 7 \\
100 \cdot 0\end{array}$ & $\begin{array}{l}24 \cdot 1 \\
25 \cdot 0 \\
50 \cdot 0 \\
47 \cdot 4 \\
58 \cdot 3\end{array}$ & $\begin{array}{l}\overline{45 \cdot 8} \\
57 \cdot 4 \\
65 \cdot 1 \\
59 \cdot 1\end{array}$ & $\begin{array}{l}27 \cdot 8 \\
46 \cdot 9 \\
52 \cdot 0 \\
68 \cdot 4 \\
66 \cdot 7\end{array}$ \\
\hline $\begin{array}{l}0^{*} \\
1 \\
2 \\
3 \\
4\end{array}$ & $\begin{array}{l}\overline{66 \cdot 7} \\
48 \cdot 6 \\
72 \cdot 4 \\
77 \cdot 3\end{array}$ & $\begin{array}{l}11 \cdot 6 \\
26 \cdot 2 \\
36 \cdot 6 \\
50 \cdot 0 \\
56 \cdot 2\end{array}$ & $\begin{array}{c}\text { Rurc } \\
-\overline{94 \cdot 4} \\
95.3 \\
96 \cdot 6 \\
100\end{array}$ & $\begin{array}{l}16 \cdot 3 \\
35 \cdot 7 \\
61 \cdot 0 \\
54 \cdot 2 \\
81 \cdot 2\end{array}$ & $\begin{array}{l}5 \overline{50} \\
34.9 \\
51 \cdot 7 \\
81.8\end{array}$ & $\begin{array}{l}30 \cdot 2 \\
47 \cdot 6 \\
56 \cdot 1 \\
54 \cdot 2 \\
75 \cdot 0\end{array}$ \\
\hline
\end{tabular}

TABLE V.-Enteroviruses Isolated from Subjects in the 1960 Survey

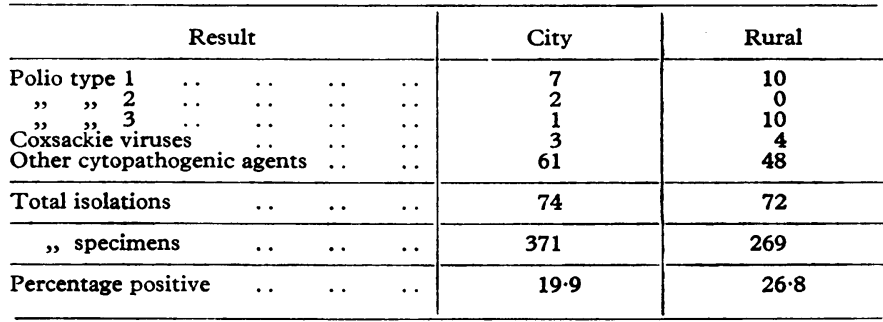

Some interesting conclusions may be drawn from Table IV. Vaccination with type 2 vaccine confers protection on a high percentage of vaccinees, and this, together with presumably naturally acquired immunity, results in protection of $100 \%$ of children 4 years old.

The rates of natural infection by all three types of polioviruses are approximately the same, all age-groups acquiring antibodies to over $50 \%$ for all three types by the age of 5 . The recent epidemic due to type 1 poliovirus did not result in a higher antibody rate for this type than for the other poliovirus types.

A minor peculiarity is the higher levels of type 1 antibody in rural vaccinees (with type 2 vaccine) as compared with nonvaccinees. A possible explanation is that in the rural areas those who came forward for vaccination were biased in favour of those who had been in contact with polio cases and had had a greater opportunity to acquire type 1 antibody by natural infection.

As may be expected, the rate of natural enterovirus circulation is higher in younger children, and higher in rural than in urban children. The overall rate of over $20 \%$ (see Table $\mathrm{V}$ ) is somewhat higher than was thought likely. It might be expected that this percentage of children in Singapore who receive live poliovaccine would be excreting some enterovirus which could cause failure of immunization due to interference.

The prevalence of type 1 and type 3 viruses was demonstrated, although the number recovered was small (28 out of 640 , or $4.5 \%$ ). Only two children were found to be excreting type 2 virus.

\section{Vaccination Campaign}

The trend in notifications of poliomyelitis cases after the epidemic of 1958 showed a new epidemic peak two years later (see Fig. 1). Also, the routine typing of cases showed that type 1 virus continued to be the main culprit, with type 3 of secondary importance (see Table VI). It was therefore evident that the pattern of endemicity with periodic epidemic peaks was likely to persist unless adequate preventive measures by vaccination were adopted. The age-pattern of cases (see Fig. 2) indicated that such measures should be directed specifically at the very young children who were most at risk. For mass vaccination of the public it was considered that only oral vaccine was practicable. The vaccine was offered on a voluntary basis through the maternal and child health centres to children 5 years of age and below.

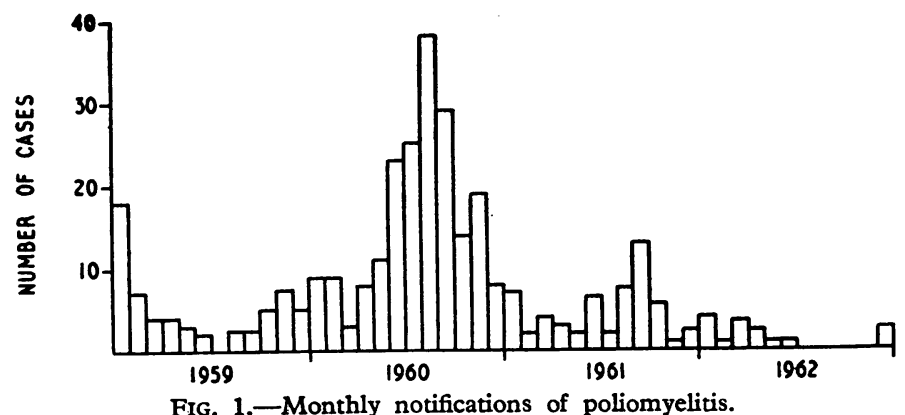


Trivalent vaccine was used in preference to monovalent vaccine in three doses. The Medical Research Council trials (P.H.L.S., 1961) had indicated that three doses of trivalent vaccine would give results as good as three doses of different monovalent vaccines. The employment of only one trivalent

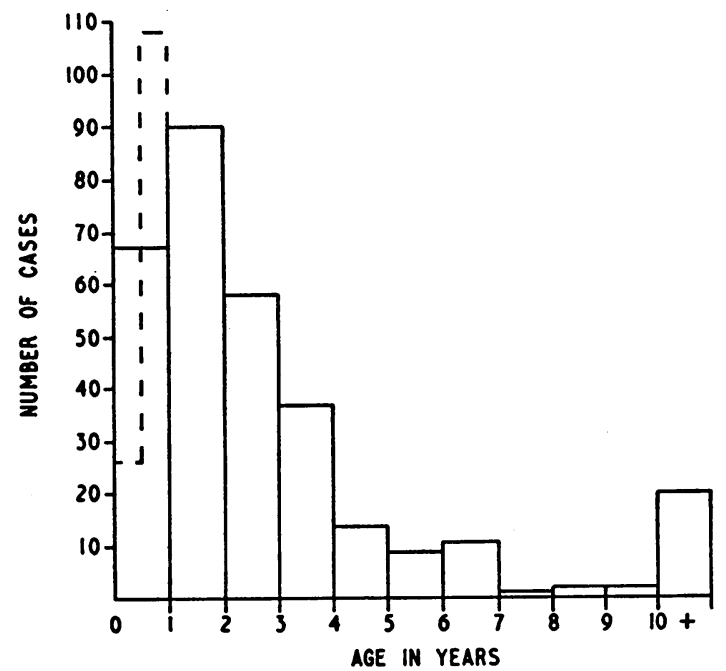

FIG. 2.-Age distribution of notified cases of poliomyelitis 1959-61.

TABLE VI.-Poliovirus Types Isolated from Notified Cases of Poliomyelitis. 1959 to 1961

\begin{tabular}{|c|c|c|c|c|c|c|c|}
\hline \multicolumn{4}{|c|}{ Result } & \multirow{2}{*}{$\begin{array}{c}1959 \\
19 \\
22 \\
11 \\
6 \\
22 \\
2\end{array}$} & \multirow{2}{*}{$\begin{array}{r}1960 \\
111 \\
1 \\
15 \\
8 \\
59 \\
2\end{array}$} & \multirow{2}{*}{$\begin{array}{r}1961 \\
11 \\
3 \\
8 \\
8 \\
20 \\
3\end{array}$} & \multirow{2}{*}{$\begin{array}{r}\text { Total } \\
141 \\
6 \\
34 \\
22 \\
101 \\
7\end{array}$} \\
\hline $\begin{array}{c}\text { Type } 1 \\
\# 3 \\
\Rightarrow 3 \\
\text { Other cyto } \\
\text { Negative } \\
\text { No specim }\end{array}$ & $\begin{array}{l}\ddot{y} \\
\ddot{a} \\
\text { opathog } \\
\text { nen }\end{array}$ & $\begin{array}{cc}\because & \cdots \\
\because & \because \\
\text { enic agents } \\
\because . & \because \\
\cdots & \cdots\end{array}$ & $\begin{array}{l}\because \\
\because \\
\because \\
\because \\
.\end{array}$ & & & & \\
\hline \multicolumn{3}{|c|}{ Total notified case } & .. & 62 & 196 & 53 & 311 \\
\hline
\end{tabular}

vaccine preparation certainly had administrative advantages. Naturally occurring enteroviruses would cause failure of immunization in some children, and it was hoped that repeated vaccination with trivalent vaccine might overcome this. However, it was decided that only two doses would be given in this campaign, and the need for a third dose would be considered after the response to two doses had been assessed.

Concentrated live poliovaccine was imported by air packed in dry ice and was stored at $-20^{\circ} \mathrm{C}$. until used. The vaccine was diluted in buffer provided plus $10 \%$ cane sugar and distributed in medicinal dropper-bottles. The dose was so adjusted that two drops would contain $10^{5.3}$ T.C.D. ${ }_{50}$ of each type. The volume delivered by the droppers was found to vary by less than $10 \%$.

Diluted vaccine was returned to the freezer and stored at $-20^{\circ}$ C. until distributed, packed in dry ice, to inoculation centres. After thawing, diluted vaccine was used up within one day, during which the thawed vaccine was kept in domestic refrigerators.

Two drops of diluted trivalent vaccine, floated on syrup in a spoon, were given to each child. The date of birth, sex, and address of each vaccinee were recorded on a form, of which the vaccinee retained a duplicate as a reminder for further doses. For administrative reasons inoculation was also recorded on the birth certificates.

The vaccination campaign began in March 1962, and by the end of June 211,776 children had received one dose of trivalent vaccine. Vaccinees were requested to return for the second dose three months later. By the end of November 1962205,000 children had received two doses. About $90 \%$ of these children were from 1 to 5 years of age ; they constituted a vaccination coverage of approximately $60 \%$ of children of this age-group in Singapore, the coverage being fairly consistent throughout this age-range.

\section{Serological Response}

Of some concern was the question whether two doses of trivalent vaccine given in the circumstances described would confer adequate protection. An attempt was made to answer this question by a study of the immune response of triplenegative children. Of 100 children between 6 and 11 months of age, from whom blood samples were taken prior to the first dose, 68 had no antibody for all three poliovirus types in the pre-inoculation serum specimen. They were given two doses of trivalent vaccine three months apart, and serum samples were obtained one month after the second dose. Of these 68 children originally triple-negative, 34 (50\%) developed neutralizing antibody for type 1 poliovirus, $67(98.5 \%)$ for type 2 , and $40(58.8 \%)$ for type 3 . In $24(35.3 \%)$ of these children there was response to only one poliovinus type-to type 2 . In $15(22 \%)$ there was response for two types, while $29(42.7 \%)$ developed antibody for all three.

The results were not surprising. Type 2 dominance in trivalent vaccine is clearly demonstrated. The response to both type 1 and type 3 was not that which could be regarded as adequate for prevention of epidemics of poliomyelitis, for which at least $80 \%$ coverage had been thought desirable.

The most encouraging aspect of the vaccination programme of 1962, however, was the dramatic fall in the incidence of paralytic poliomyelitis (see Table II and Fig. 1). It is possible that some other factors may be responsible for the marked drop in incidence at the end of 1962, but it is not unreasonable to infer that this was actually due to vaccination. That a two-dose campaign might have yielded such a result is a happy unforeseen event.

\section{Current Programme for Prevention of Poliomyelitis}

On the basis of the experience gained, the Government of Singapore in 1963 inaugurated a routine programme of immunization on a voluntary basis with oral vaccine.

The inclusion of type 2 virus in trivalent vaccine undoubtedly depressed the response to the other types. A $100 \%$ response to type 2 is somewhat of an unnecessary luxury since the incidence of type 2 cases has always been comparatively low. For this reason the course of immunization was altered to two doses of bivalent vaccine comprising types 1 and 3 followed by one dose of trivalent vaccine. Magnesium chloride was added to the vaccine at one molar concentration as stabilizer (Wallis and Melnick, 1961).

Since 1963 this schedule of poliomyelitis immunization has been offered to children in the first year of life, when they turn up for vaccination against diphtheria. (Diphtheria immunization was recently made compulsory, and requires three doses of diphtheria vaccine, so advantage is taken of the three visits to give polio vaccine as well). The children are usually about 3 to 4 months old at the first visit, and although the antibody response of such young infants may be relatively poor, it is desirable to try to protect them against poliomyelitis since there is already a significant case incidence between the ages of 6 months and one year (see Fig. 2).

Concurrently, children attending school for the first time at about 7 years of age are also being offered polio vaccine. If any of these children had previously been vaccinated they would be given only a so-called reinforcing course of one dose of bivalent and one dose of trivalent vaccine. Opportunity for polio immunization in the schools is provided by the school health authorities' existing programme of school visits for the purpose of giving diphtheria vaccine. 
It is confidently expected that the current programme will lead to a permanent change in the incidence of poliomyelitis in Singapore.

\section{Discussion}

Singapore presents certain features which make it a favourable locale for epidemiological studies in the prevention of poliomyelitis. The health services and notification coverage are sufficiently well developed to ensure that cases of paralytic poliomyelitis come to attention, and laboratory facilities are available for virological studies.

The attack rate of poliomyelitis has been high and cases have occurred sporadically and in epidemics. Oral vaccination has been adopted by the health authorities for the prevention of the disease. The first phase of this programme was carried out in 1962, when a large proportion of children from 6 months to 5 years of age were given two doses, about three months apart, of trivalent oral vaccine.

The immune response of triple-negative infants to two doses of trivalent vaccine was not satisfactory, since only about $50 \%$ developed antibody for type 1 and type 3 poliovirus. However, it has been shown that children of older age-groups had high antibody rates for all poliovirus types prior to vaccination, and the two-dose campaign may be regarded as having improved the immune status of the child population considerably. That the incidence of poliomyelitis in the second half of 1962 was practically nil may very well have been a consequence of the vaccination campaign.

In 1963 the second phase of the vaccination programme was inaugurated. Children in the first year of life receive three doses of oral vaccine-the first two of bivalent (types 1 and 3) and the last of trivalent vaccine. This will be the principal measure for the control of poliomyelitis in Singapore. As a supplementary measure, children attending school for the first time will be given the primary course of three doses if they had never received the vaccine before, or a " reinforcement" course if they had had two doses in the 1962 campaign. It remains to be seen whether the "routine" programme of primary immunization of the newborn and reinforcement of children in their first school year-for example, at about 7 years of age-will keep the incidence at a satisfactorily low level.

When a "vaccine trial" is conducted it may be expected that special efforts are made to provide the optimum conditions in regard to the distribution and administration of the vaccine. When the vaccine is put into "routine" use on a permanent basis, administrative procedures have to be reduced to a reasonable minimum. The effectiveness of a vaccine should be assessed under the conditions in which it is employed in practice. In this regard, the oral-vaccine programme in Singapore is a long-term experiment on the routine use of this vaccine. It is hoped that over the next few years our observations may cast light on a number of questions.

It has to be ascertained that the schedule of dosage for primary immunization with types 1 and 3 twice, followed by trivalent vaccine, does indeed give adequate protection. The deferment of immunization with type 2 vaccine until the last dose seems to be sound in view of its interfering properties and the low incidence of type 2 cases. It is possible that the latter circumstance may change, necessitating an alteration in the dosage schedule. Bivalent and trivalent vaccines were adopted because of the high rate of naturally occurring enteroviruses. It seems reasonable to try to overcome a possible high rate of interference from naturally occurring enterovirus by repeated doses of vaccine virus types 1 and 3 , the types epidemiologically important, in spite of the added costs when compared with a course of monovalent vaccines each given once.
Primary immunization at the early age of 3 months was adopted because a fair proportion of cases occurred before the age of 1 year. It is well known, however, that the immune response of young infants is poor, and whether the protection conferred at infancy would persist until the children go to school, where they receive the reinforcement doses, remains to be seen. Whether these immunizations on two occasions in childhood would confer protection for life is a question that cannot yet be answered.

The serological survey that was carried out before the introduction of routine immunization is a valuable baseline for future studies of immune status of the population of Singapore. Careful documentation of those receiving the vaccine should permit their accurate identification when future serological surveys are conducted.

The cost of the vaccination programme has not been inconsiderable. In terms of the saving of human suffering by preventing poliomyelitis, the expense of the programme could probably be justified. If it achieves a large saving in the previously high cost of the care of poliomyelitis patients this should more than offset the cost of the programme. This saving will be apparent when the authorities are able to reduce the provision of hospital beds for poliomyelitis patients. The poliomyelitis-prevention programme in Singapore would then have attained its objective.

\section{Summary}

A serological survey in 1960 of 641 children in Singapore 18 months after an extensive trial of Sabin-type 2 oral vaccine showed that children have high antibody rates for three polio types by the age of 5 years. A faecal survey conducted at the same time showed a high incidence of naturally occurring enteroviruses.

The incidence of paralytic poliomyelitis is greatest about the age of 1 year. Type 1 poliovirus is responsible for most cases, type 3 is a minor offender, and type 2 is comparatively rare.

Over 200,000 children between the ages of 6 months and 6 years received two doses of trivalent oral vaccine in 1962. The number vaccinated represents approximately $60 \%$ of children from 1 to 5 years of age. As a probable consequence the incidence of paralytic poliomyelitis during the latter part of 1962 dropped to practically nil.

In 1963 a programme of primary immunization of infants with two doses of bivalent vaccine (types 1 and 3) and one dose of trivalent vaccine has been introduced. Children about 7 years old, in the first year at school, were given either primary immunization or a reinforcement schedule of one bivalent and one trivalent dose.

The practical problems of a long-term programme of immunization are briefly discussed.

We are grateful to the Director of Medical Services for permission to quote from official documents of the Poliomyelitis Committee. It is a pleasure to acknowledge the helpful collaboration of the staff of the Singapore Government Medical Department, especially Dr. K. W. Leong, acting superintendent, Middleton (infectious diseases) Hospital ; of Dr. M. Lim, medical officer in charge Maternity and Child Welfare Department, and of the other doctors and nurses concerned with the poliomyelitis vaccination programme. We also wish to acknowledge the co-operation of those members of the public who were the subjects of the investigations reported.

\section{REFERENCES}

Hale, J. H., Lee, L. H., Doraisingham, M., Kanagaratnam, K., Leong, Hale, J. H., Lee, L. H., Doraisingham, M., Kanagaratnam, K., Wallis, C., and Melnick, J. L. (1961). Tex. Rep. Biol. Med., 19, 683. W.H.L.S. (1961). Brit. med. Ұ., 2, 1037.

Ministry of Health, Singapore (1961). Report of Committee on Poliomyelitis. 